\title{
ON q-TH DERIVATIVE OF VECTOR BUNDLES
}

\author{
AKIKUNI KATO
}

To Professor Kiyoshi Noshiro on the occasion of his 60th birthday

(0.0) In the present note we shall be concerned with the improvement of fundamental definitions in higher order enumerative geometry which has been recently given by W. F. Pohl. Pohl's definition of $q$-th derivative of vector bundle is very complicated. We shall give a simpler and more reasonable definition of the $q$-th derivative of vector bundle in terms of sheaf theory and simplify the proofs in [P]. We shall also give a definition of higher order singularity of map.

\section{The sheaf $D_{q}$}

(1.1) Let $\left(X, O_{x}\right)$ be an object of a category $C$ of ringed spaces over ground field $k$. Ringed space over $k$ is a pair $\left(X, O_{X}\right)$ of topological space $X$ and sheaf $O_{X}$ of $k$-algebras over $X$. (cf. [G]).

The following are most important examples of the category $C$;

(i) the category of differentiable-manifolds; $k$ is the field $R$ of real numbers.

(ii) the category of complex manifolds; $k$ is the field $\mathbf{C}$ of complex numbers.

(iii) the category of algebraic $k$-schemes (or varieties defined over $k$ ).

(1.2) Let $F_{1}$ be the sheaf of derivations of $O_{X}$ over $k$, i.e.

$$
\begin{aligned}
F_{1}=\left\{t \in \operatorname{Hom}_{k}\left(O_{x}, O_{x}\right) \mid t(a b)=a \cdot t(b)+b t(a)\right. & \text { for any } a, b \in O_{x} \\
& \text { and } t(c)=0 \text { for any } c \in k\}
\end{aligned}
$$

where $\operatorname{Hom}_{k}\left(O_{x}, O_{X}\right)$ denotes the sheaf of $k$-homomorphisms of $O_{x}$ into itself.

Then, $F_{1}$ is a sheaf of $k$-modules. Tensoring $O_{x}$ with $F_{1}$ over $k$, we get a sheaf of $O_{X}$-modules $F=F_{1} \otimes_{k} O_{X}$.

Although we may also consider $F_{1}$ as a sheaf of $O_{x}$-modules, we do not

Received May 23, 1966.

The author wishes to extend his hearty gratitude to Professor H. Morikawa under whose direction this paper was prepared. 
give $O_{x}$-modules structure to $F_{1}$. Hence $O_{x}$-modules structure of $F$ is given only by base extension.

(1.3) Let $T(F)$ be a tensor algebra of $F$ over $O_{x}$, i.e. $T(F)=\bigoplus_{n \geq 0} F^{\otimes^{n}} . \quad T(F)$ is a sheaf of graded rings. Let $p$ be a characteristic of a field $k$. Then, $\mathfrak{A}$ is a sheaf of two-sided ideals of $T(F)$ generated by the elements :

$t \otimes a-a t \otimes 1-t(a),\left(t_{1} \otimes 1\right) \otimes\left(t_{2} \otimes 1\right)-\left(t_{2} \otimes 1\right) \otimes\left(t_{1} \otimes 1\right)-\left[t_{1}, t_{2}\right] \otimes 1,(t \otimes 1)^{\otimes p}$ $-t^{p}$, where $t, t_{1}, t_{2} \in F_{\mathrm{t}}, a \in O_{x}$ and $\left[t_{1}, t_{2}\right]$ denotes Lie bracket of $t_{1}$ and $t_{2}$.

The quotient $D$ of $T(F)$ by $\mathfrak{A}$ is a sheaf of filtered rings. If we denote by $D_{q}$ the $q$ th part of $D$, then $D_{0}=O_{x}$, and we put $D_{q} / D_{0}=F_{q}$ which is called the sheaf of germs of osculating vector fields of order $q$ in $[\mathrm{P}]$. We denote by $t_{1} a_{1} t_{2} a_{2} \cdots t_{q} a_{q}$ the canonical image in $D$ of $\left(t_{1} \otimes a_{1}\right) \otimes\left(t_{2} \otimes a_{2}\right) \otimes \cdots \otimes\left(t_{q} \otimes a_{q}\right)$ and $t \cdot 1=1 \cdot t$ is also written simply by $t$.

(1.4) Let $\mathbf{S}\left(F_{1}\right)$ be a symmetric algebra of $F_{1}$ over $k$, i.e. $\mathbf{S}\left(F_{1}\right)$ is the quotient of tensor algebra $T\left(F_{1}\right)$ by the ideal $\left\{t \otimes u-u \otimes t / t, u \in F_{1}\right\}$. Then $\mathbf{S}\left(F_{1}\right)$ is a sheaf of graded rings. We denote by $\mathbf{S}^{7}\left(F_{1}\right)$ the $q$-th degree part of $\mathbf{S}\left(F_{1}\right)$. There is a canonical homomorphism

$$
S_{q}: F_{1}^{\otimes a} \rightarrow \mathrm{S}^{q}\left(F_{1}\right) .
$$

A homomorphism $m: F_{1}^{\otimes q} \rightarrow D_{q}$ is defined by $\left.m_{1}^{\prime} t_{1} \otimes t_{2} \otimes \cdots \otimes t_{q}\right)=t_{1} t_{2} \cdots t_{q}$. Then for $q \geq 1$, we have $m\left(t_{1} \otimes t_{2} \otimes \cdots \otimes t_{q}-t_{1} \otimes \cdots \otimes t_{i+1} \otimes t_{i} \otimes \cdots \otimes t_{q}\right)=$ $t_{1} \cdots t_{i-1}\left[t_{i}, t_{i+1}\right] t_{i+2} \cdots t_{q} D_{q-1}$, hence $m$ induces a homomorphism

$$
\varphi_{q}: \mathbf{S}^{q}\left(F_{1}\right) \rightarrow D_{q} / D_{q-1} \text { for } q \geq 1 \text {. }
$$

Proposition (1.5) $\varphi_{a}$ is an isomorphism, hence we have an exact sequence

$$
0 \longrightarrow D_{q-1} \stackrel{I_{q}}{\longrightarrow} D_{q} \longrightarrow \mathbf{S}^{\urcorner}\left(F_{1}\right) \longrightarrow 0
$$

where $I_{q}$ is a canonical inclusion.

Proof (cf. [P]. Th. 2.1)

Corollary (1.6) If $F_{1}$ is locally free, then $D_{q}$ is also locally free.

Proof. If $F_{1}$ is locally free, then $\mathbf{S}^{q}\left(F_{1}\right)$ is locally free and it is proved by induction on $q$.

\section{The sheaf $\Delta_{q} \mathrm{M}$}

Definition (2.1) Let $M$ be a sheaf of $O_{x}$-modules. The sheaf $\Delta_{q} M=D_{q} \otimes o_{X} \dot{M}$ 
is called the $q$-th derivative of $M$.

It is easily verified that this definition of $q$-th derivative of $M$ is the same as Pohl's one (cf. [P]. $\S$ III. 1.). $\Delta_{q}$ is a functor from the category of sheaves of $O_{x}$-modules into itself.

Assumption (2.2) Assume that in category $C, F_{1}$ is always locally free. This assumption is satisfied, for example, if $C$ is (i), (ii) of (1.1) or the category of non-singular algebraic varieties.

Proposition (2.3) Under the assumption (2.2), there is an exact sequence for $q \geq 1$,

$$
0 \longrightarrow \Delta_{q-1} M \stackrel{I_{q}^{M}}{\longrightarrow} \Delta_{q} M \longrightarrow \mathrm{S}^{q}\left(F_{1}\right) \otimes o_{X} M \longrightarrow 0
$$

where $I_{q}^{M}=I_{q} \otimes i_{. u}$ and $i_{M}$ is identity of $M$.

Proof. By (1.5.1), there is an exact sequence

$$
0 \rightarrow D_{q-1} \rightarrow D_{q} \rightarrow \mathbf{S}^{q}\left(F_{1}\right) \rightarrow 0 .
$$

Tensoring $M$, we get an exact sequence

$$
D_{q-1} \otimes M \rightarrow D_{q} \otimes M \rightarrow \mathbf{S}^{q}\left(F_{1}\right) \otimes o_{X} M \rightarrow 0 .
$$

On the other hand, $\mathbf{S}^{q}\left(F_{1}\right)$ is locally free, and so is $O_{X}$-flat. Therefore (2.3.1) is exact (cf. [B] I, 2 Prop. 4).

Proposition (2.4) Under the assumption (2.2), the exact sequence (2.3.1) is an exact functor of $M$. In particular $\Delta_{q}$ is an exact functor.

Proof. If $F_{1}$ is locally free, $D_{q}$ and $\mathbf{S}^{q}\left(F_{1}\right)$ are also locally free.

(2.5) We define a homomorphism $\varphi_{1}: F=F_{1} \otimes_{k} O_{X} \rightarrow O_{X}$ by $\varphi_{1}(t \otimes a)=t(a)$, $t \in F_{1}, a \in O_{x} . \quad$ A homomorphism $\varphi_{n}: F^{\otimes n} \rightarrow O_{x}$ is defined to be $\varphi_{n}=\varphi_{1} \circ\left(i_{F} \otimes \varphi_{n-1}\right)$ where $i_{F}$ is identity homomorphism of $F$. Let $\varphi_{0}: O_{x} \rightarrow O_{x}$ be the identity. From $\left\{\varphi_{n}\right\}_{n=0}$, we get a homomorphism

$$
\varphi: T(F) \rightarrow O_{X} .
$$

It is clear that $\operatorname{Ker} \varphi \supset \mathfrak{A}$. $\varphi$ induces a homomorphism

$$
\rho: D=T(F) / \mathfrak{R} \rightarrow O_{x} .
$$

We denote by $\rho_{q}$ the restriction of $\rho$ on $D_{q}$, i.e. $\rho_{q}: D_{q} \rightarrow O_{x}$. Since $D_{q} \otimes \rho_{x} O_{x}$ $=D_{q}, \Delta_{q} O_{x}^{N}=D_{y}^{N}$. 
Definition (2.5.1) We denote by $\omega_{q}$ the map $\rho_{q}^{N}=\rho_{q}+\cdots+\rho_{q}: \Delta_{q} O_{X}^{N} \rightarrow O_{X}^{N}$. The restriction of $\omega_{q}$ on $\Delta_{q-1} O_{x}^{N}$ is $\omega_{q-1}$, i.e.

$$
\omega_{q-1}=\omega_{q} \circ I_{q}^{O_{X}^{N}}
$$

because it holds $\rho_{q-1}=\rho_{q} \circ I_{q}$ (cf. [P]. Prop. 3. $9_{q}$ )

Let $\omega_{0}: \Delta_{0} O_{X}^{N}=O_{x}^{N} \rightarrow O_{X}^{N}$ be the identity. We get also

$$
\omega_{1} \circ I_{0}^{O_{X}^{N}}=\text { identity, }
$$

i.e. $\omega_{1}$ defines a splitting of (2.3.1) for $q=1$ (cf [P]. Prop. 3. 8).

\section{Higher order singularities of maps}

(3.1) Let $G_{n, r}$ be the Grassmanian manifold of $(r-1)$-dimensional projective subspaces in $n$-dimensional projective space. Given a suitable structure sheaf $O_{G}$ on $G_{n, r}, G_{n, r}$ may be considered as an object of category (i), (ii) or (iii) of (1.1). $G_{n, r}$ is imbedded in some projective space by Plücker coordinates $p=\left(\ldots, p_{i_{1} \ldots i_{r}}, \ldots\right)$. Plücker coordinates $p_{i_{1} \ldots i_{r}}$ satisfy the well-known relations

$$
\sum_{k=1}^{r+1}(-1)^{k} p_{i_{1} \ldots i_{r-2}} j_{k} p_{j_{1} \ldots \hat{j}_{k} \ldots j_{r+1}}=0
$$

for any $i_{1}, \ldots, i_{r-1}, j_{1}, \ldots, j_{r+1}$ such that $1 \leq i_{1}<\cdots<i_{r-1} \leq n, 1 \leq j_{1}<\cdots$ $<j_{n+1} \leq n$.

(3.2) Let $V_{i_{1} \ldots i_{r}}$ be the set of points $p=\left(\ldots, p_{i_{1} \ldots i_{r}}, \ldots\right)$ of $G_{n, r}$ such that $p_{i_{1} \ldots i_{r}} \neq 0$ Then $V_{i_{1} \ldots i_{r}}$ is an (affine) open subset of $G_{n, r}$, and $\left\{V_{i_{1} \ldots i_{r}}\right\}$ is an open covering of $G_{n, r}$. Let $\mathscr{A}_{i_{1} \ldots i_{r}}$ be the restriction of the sheaf $O_{G}^{r}$ on $V_{i_{1} \ldots i_{r}}$. Let $v_{1}^{(i)}, \ldots, v_{r}^{(i)}$ and $v_{1}^{(j)}, \ldots, v_{r}^{(j)}$ be the canonical basis of $\mathscr{A}_{i_{1} \ldots i_{r}}$ and $\mathscr{A}_{j_{1} \ldots j_{r}}$ respectively. Now we define a homomorphism

(3. 2.1) $f_{(j)}^{(i)}=f_{j_{1} \ldots j_{r}}^{i_{1} \ldots i_{r}}: \mathscr{A}_{i_{1} \ldots i_{r}}\left|V_{i_{1} \ldots i_{r}} \cap V_{j_{1} \ldots j_{r}} \rightarrow \mathscr{A}_{j_{1} \ldots j_{r}}\right| V_{i_{1} \ldots i_{r}} \cap V_{j_{1} \ldots j_{r}}$,

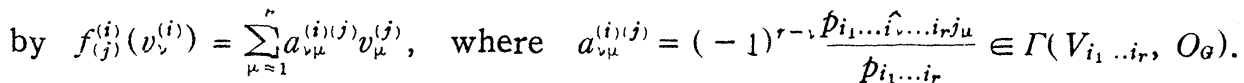

We shall show $f_{(k)}^{(j)} f_{(j)}^{(i)}=f_{(k)}^{(i)}$ on $V_{i_{1} \ldots i_{r}} \cap V_{j_{1} \ldots j_{r}} \cap V_{k_{1} \ldots k_{r}}$ for any $i_{1}, \ldots, i_{r}$, $j_{1}, \ldots, j_{r}, k_{1}, \ldots, k_{r}$.

$$
\begin{aligned}
f_{(k)}^{(j)} f_{(j)}^{(i)}\left(v_{\nu}^{(i)}\right) & =\sum_{\mu=1}^{r} a_{\nu \mu}^{(i)(j)} f_{(k)}^{(j)}\left(v_{\mu}^{(j)}\right) \\
& =\sum_{i=1}^{r} a_{\nu \lambda}^{(i)(j)}\left(\sum_{\lambda=1}^{r} a_{\mu \lambda}^{(j)(k)} v_{\Lambda}^{(k)}\right)=\sum_{\Lambda=1}^{r}\left(\sum_{\mu=1}^{r} a_{\nu_{\mu}}^{(i)(j)} a_{\mu \lambda}^{(j)(k)}\right) v_{\Lambda}^{(k)}
\end{aligned}
$$




$$
\begin{aligned}
& =\sum_{\lambda=1}^{r} \frac{(-1)^{r-\nu}}{p_{i_{1} \ldots i_{r}} \cdot p_{j_{1} \ldots j_{r}}}\left(p_{i_{1} \ldots \hat{i}_{\nu} \ldots i_{r} j_{1}} \cdot(-1)^{r-1}\right. \\
& p_{j_{2} \ldots j_{r} k_{\curlywedge}}+p_{i_{1} \ldots \hat{i}_{\nu} \ldots i_{r} j_{:}}(-1)^{r-2} p_{j_{1} j_{3} \ldots j_{r} k_{\lambda}}+ \\
& \left.\cdots+p_{i_{1} \ldots \hat{i}_{2} \ldots i_{r} j_{r}} \cdot p_{j_{1} \ldots i_{r-1} k_{\lambda}}\right) v_{\lambda}^{(k)} \\
& =\sum_{\lambda=1}^{r} \frac{(-1)^{r-\nu} p_{i_{1}} \ldots \hat{i}_{2} \ldots i_{r} k_{\lambda} \cdot p_{j_{1} \ldots j_{r}}}{p_{i_{1} \ldots i_{r}} \cdot p_{j_{1} \ldots j_{r}}} v_{\lambda}^{(k)} \\
& =\sum_{\lambda=1}^{r} a_{\nu \mu}^{(i)(k)} v_{\lambda}^{(k)}=f_{(k)}^{(i)}\left(v_{(\nu)}^{(i)}\right) \text {. }
\end{aligned}
$$

It is clear that $f_{(i)}^{(i)}=$ identity, hence $f_{(j)}^{(i)}=\left(f_{(i)}^{(j)}\right)^{-1}$ on $V_{i_{1} \ldots i_{r}} \cap V_{j_{1}, \ldots, j_{r}}$, i.e. the homomorphism $f_{(j)}^{(i)}$ of $(3.2 .1)$ is an isomorphism. Then we can patch $\mathscr{A}_{i_{1} \ldots i_{r}}$ together with the isomorphism (3.2.1) and we obtain a sheaf of $O_{G}$-modules $\mathscr{E}_{n, r}$ over $G_{n, r}$ (cf [G] Chap. 0, (3.3.1)). $\mathscr{E}_{n, r}$ is locally free of rank $r$.

Remark (3.2.2) $\mathscr{E}_{n, r}$ is the sheaf of germs of cross-sections of the universal $r$-plane bundle over $G_{n, r}$.

(3.3) We shall define a homomorphism $\sigma$ of $\mathscr{C}_{n, r}$ into $O_{G}^{n+1}$. Firstly we define a homomorphism

$$
\sigma_{i_{1} \ldots i_{r}}: \mathscr{A}_{i_{1} \ldots i_{r}} \simeq \mathscr{E}_{n, r}\left|V_{i_{1} \ldots i_{r}} \rightarrow O_{G}^{n+1}\right| V_{i_{1} \ldots i_{r}}
$$

Let $u_{0}, \ldots, u_{n}$ be the canonical basis of $O_{G}^{n+1}$. Then $\sigma_{i_{1} \ldots i_{r}}$ is defined by $\sigma_{i_{1} \ldots i_{r}}\left(v_{\nu}^{(i)}\right)=\sum_{\mu=0}^{n} b_{\nu \mu} \cdot u_{\mu}$ where $b_{\nu \mu}=(-1)^{r-\nu} \frac{p_{i_{1} \ldots \hat{i}_{2} \ldots i_{r}}}{p_{i_{1} \ldots i_{r}}} \in \Gamma\left(V_{i_{1} \ldots i_{r}}, O_{G}\right)$.

By virtue of (3.1.1), it is easily verified that

$$
\sigma_{i_{1} \ldots i_{r}}\left|V_{i_{1} \ldots i_{r}} \cap V_{j_{1} \ldots j_{r}}=\sigma_{j_{1} \ldots j_{r}}\right| V_{i_{1} \ldots i_{r}} \cap V_{j_{1} \ldots j_{r}} .
$$

Patching $\sigma_{i_{1} \ldots i_{r}}$ together, we get a homomorphism

$$
\sigma: \mathscr{E}_{n, r} \rightarrow O_{\Theta}^{n+1} \text {. }
$$

(3.4) Let $f$ be a morphism of $X$ into $G_{n, r}$. By virtue of the homomorphism $\sigma$ in (3.3.1), we obtain a homomorphism

$$
f^{*}(\sigma): f^{*}\left(\mathscr{E}_{n, r}\right) \rightarrow O_{x}^{n+1}=f^{*}\left(O_{\xi}^{n+1}\right)
$$

(where $f^{*}$ denotes the reciprocal image) and consequently a homomorphism

$$
\Delta_{q} f^{*}(\sigma): \Delta_{q} f^{*}\left(\mathscr{C}_{n, r}\right) \rightarrow \Delta_{q}\left(O_{X}^{n+1}\right) .
$$

Definition (3.5) We say that the morphism $f: X \rightarrow G_{n, r}$ is non-singular of oraer $q$ at $x \in X$, if the homomorphism

$$
\omega_{q} \circ \Delta_{q} f^{*}(\sigma): \Delta_{q} f^{*}\left(\mathscr{C}_{n, r}\right) \rightarrow O_{X}^{n+1},
$$


which is the composition of $A_{q} f^{*}(\sigma)$ of $(3.4 .1)$ and $\omega_{q}$ of $(2.5 .1)$, is injective at $X$.

\section{REFERENCES}

[B] N. Bourbaki: Algèbre commutative. chap. 1, 2.

[G] A. Grothendieck: Éléments de géométrie algébrique. Publ. Math. I.H.E.S.

[P] W. F. Pohl: Differential geometry of higher order. Topology I, 169-211, 1962. 